Airborne Astronomy Program

Medium Altitude Missions Branch

Preprint Series 042

NASA-TM-86851

19860008807

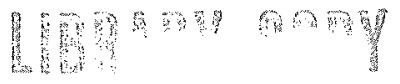

MMPUH

\title{
Observations of the $\mathrm{J}=10$ Manifold of the Pure Rotational Band of Phosphine on Saturn
}

M.R. Haas, E.F. Erickson, D. Goorvitch, D.D. McKibbin, and D.M. Rank

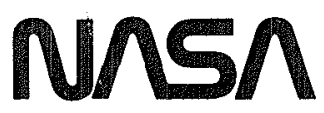

National Aeronautics and Space Administration

January 1986

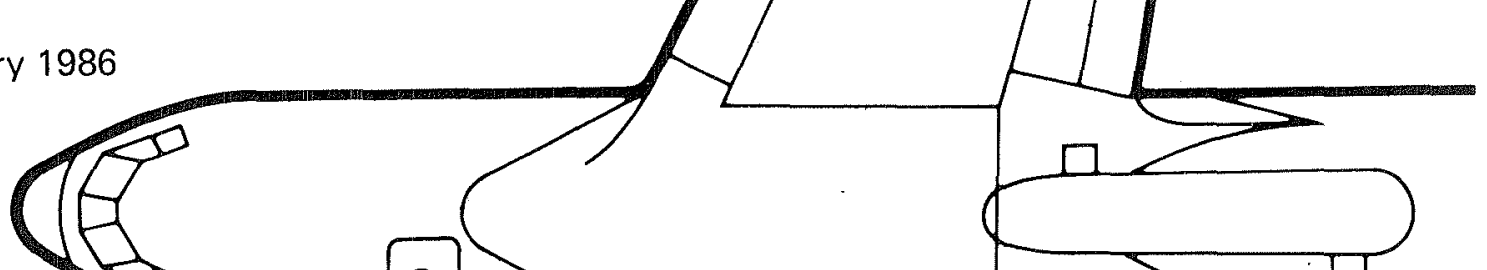




\section{Observations of the $\mathrm{J}=\mathbf{1 0}$ Manifold of the Pure Rotational Band of Phosphine on Saturn}

M. R. Haas, Mycol, Incorporated, Sunnyvale, California

E. F. Erickson,

D. Goorvitch,

D. D. McKibbin, Ames Research Center, Moffett Field, California

D. M. Rank, University of California, Santa Cruz, California

January 1986

\section{N/SA}

National Aeronautics and

Space Administration 
OBSERVATIONS OF THE $J=10$ MANIFOLD OF THE PURE ROTATIONAL BAND . OF PHOSPHINE ON SATURN

\author{
M. R. Haas \\ Mycol, Incorporated \\ 1067 Mango Avenue \\ Sunnyvale, CA 94087 \\ and
}

E. F. Erickson, D. Goorvitch, D. D. McKibbin

MS 245-6, Space Science Division

NASA-Ames Research Center

Moffett Field, CA 94035

and

D. M. Rank

Lick Observatory, University of California

Santa Cruz, CA 95064

Received:

Manuscript: 22

Figures: 5

Tables: 1 


\begin{abstract}
Saturn was observed in the vicinity of the $J=10$ manifold of the pure rotational band of phosphine on 1984 July 10 and 12 from NASA's Kuiper Airborne Observatory with the facility far-infrared cooled grating spectrometer. On each night observations of the full disk plus rings were made at 4 to 6 discrete wavelengths which selectively sampled the manifold and the adjacent continuum. The previously reported detection of this manifold is confirmed. After subtraction of the flux due to the rings, the data are compared with disk-averaged models of Saturn. It is found that $\mathrm{PH}_{3}$ must be strongly depleted above the thermal inversion ( -70 mbar). The best fltting models consistent with other observational constraints indicate that $\mathrm{PH}_{3}$ is significantly depleted at even deeper atmospheric levels ( $\$ 500$ mbar), implying an eddy diffusion coefficient for saturn of $-10^{4} \mathrm{~cm}^{2} \mathrm{sec}^{-1}$.
\end{abstract}




\section{INTRODUCTION}

The presence of phosphine $\left(\mathrm{PH}_{3}\right)$ in Saturn's troposphere has been well established by observations at near and middle infrared wavelengths (Larson et al., 1980 and references therein; Hanel et al., 1981). This was somewhat surprising because thermochemical equilibrium calculations indicated that for temperatures $\leqq 2000 \mathrm{~K}$ phosphine would react with $\mathrm{H}_{2} \mathrm{O}$ (Lewis, 1969; Barshay and Lewis, 1978). However, its presence may be explainable by some combination of modest convection, an upper atmospheric recycling mechanism, and low abundances of $\mathrm{H}_{2} \mathrm{O}$ and $\mathrm{NH}_{3}$ (Larson et al., 1980). The observed $\mathrm{PH}_{3}$ abundance implies that the $\mathrm{P} / \mathrm{H}$ ratio is approximately 3 times the solar value (Encrenaz and Combes, 1980 and references therein; Courtin et al., 1984), which may have interesting implications for cosmogony (Gautier and Owen, 1983).

Moreover, the vertical distribution of $\mathrm{PH}_{3}$ is important to our understanding of its chemistry and the vertical dynamics on Saturn (Prinn and Lewis, 1975; Strobel, 1978; Larson et al., 1980). From IUE ultraviolet spectra Winkelstein et al. (1983) concluded that there is little or no $\mathrm{PH}_{3}$ above 25 mbar, but from Voyager IRIS spectra Courtin et al. (1984) inferred a uniform mixing ratio of $3.0 \pm 0.5 \times 10^{-6}(5 \mathrm{X}$ solar) up to the $3-5 \mathrm{mbar}$ level. Given the $\mathrm{PH}_{3}$ abundance inferred at shorter wavelengths, the far infrared manifolds of the pure rotational band of $\mathrm{PH}_{3}$ are predicted to be quite strong (Encrenaz and Combes, 1977). This band is expected to reach optical depth unity near the thermal inversion ( -70 mbar), thereby probing higher levels of the atmosphere than the near and middle infrared vibrational transitions (Encrenaz and Combes, 1980). Observations of this 
band will therefore place new constraints on the $\mathrm{PH}_{3}$ distribution in the vicinity of the tropopause.

Haas et al. (1984) previously detected the $J=10$ manifold at $103 \mu \mathrm{m}$ and Viscuso $(1984)^{1}$ may have detected the $J=7$ manifold at $141 \mu \mathrm{m}$. In this paper we report new observations of the $J=10$ manifold with higher signal-to-noise and improved calibration. We also describe our ring subtraction procedure and present a number of disk-averaged theoretical models. The implications of the best fitting models are discussed.

\section{OBSERVATIONS AND DATA ANALYSIS}

Saturn was observed on the nights of $1984 \mathrm{July} 10$ and 12 with the facility far-infrared cooled grating spectrometer (CGS) on the $91 \mathrm{~cm}$ telescope of the Kuiper Airborne Observatory from an altitude of $12.0 \mathrm{~km}$. The FWHM beam size was $40^{\prime \prime}$ and the chopper throw was $44^{\prime}$. The CGS was operated with six discrete Ge:Ga detectors in the dispersed focal plane with a wavelength separation of $0.0314 \mu \mathrm{m}$ at $103 \mu \mathrm{m}$. The detectors oversampled the spectrum in wavelength by a factor of about 1.8. Additional details regarding the CGS design, performance, and typical observing procedures are described in Erickson et al. (1984a,b; 1985).

The resolution of the CGS is high enough that an entire $\mathrm{PH}_{3}$ manifold ( $-3 \mu \mathrm{m}$ wide) could not be scanned in the available observing time ( $-15 \mathrm{~min})$. Hence observations were made at 4 to 6 discrete wavelengths (grating positions) which were chosen to minimize the effects of telluric absorption (see Figure 1a) and optimize the wavelength coverage of the important spectral features. Four pairs of right-left beam integrations totaling 80 1 Private communication 
sec were taken at each wavelength. The absolute flux calibration and the relative detector response were obtained by observing Mars on the same flights. The final spectra are simply the ratio of the signals measured on Saturn to those measured on Mars and multiplied by the Mars flux corresponding to a brightness temperature of $227 \mathrm{~K}$ at all wavelengths as predicted by the model of Simpson et al. (1981). At this epoch the diameter of the Martian disk was 13.18", the Saturnian disk was $17.39^{\prime \prime} \times 15.71 "$, and the inclination of the rings was $18.99^{\circ}$ (Vohden and Boksenberg, 1984). Following Matthews and Erickson (1977), we calculated the total area of the Saturn ring-disk system to be 405.0 square arcsec. The Saturn flux was increased by $6 \%$ to account for the effects of diffraction in the telescope and geometrical losses due to the extended nature of the rings relative to the beam. No second order water vapor correction was applied because the two planets were observed at a similar elevation and aircraft heading and there are no strong telluric features at the wavelengths in question (Figure 1a).

The brightness temperatures measured for the Saturnian ring-disk system are plotted in Figure $1 \mathrm{~b}$ and listed in Table 1. A decrease in brightness temperature of $-5 \mathrm{~K}$ at the expected position of the $\mathrm{J}=10$ manifold of $\mathrm{PH}_{3}$ is clearly evident in both data sets. No other molecules are expected to produce absorption or emission features at these wavelengths; the $J=4$ manifold of $\mathrm{NH}_{3}$ (cf Figure 2) has been carefully avoided. To improve the signal-to-noise, the six detectors have been averaged at each grating position. Their total bandpass is $0.19 \mu \mathrm{m}$ and the uncertainty in the wavelength is $-0.015 \mu \mathrm{m}$. The quoted error bars are statistical errors only; they represent one-standard-deviation-of-the-mean for the six detectors. 
Systematic uncertainties will increase these errors somewhat, but are difficult to estimate. There is a $15 \%$ difference in the absolute calibration between the two nights! data. However, the good night-to-night agreement in the shape of the overall spectrum suggests that the relative calibration of the data points on a single night is significantly better.

\section{THE RING SUBTRACTION PROCEDURE}

The contrast in the $\mathrm{PH}_{3}$ line is reduced by the continuum emission of the rings. In order to directly compare our measurements with models of the disk of Saturn, we must subtract the ring contribution. To this end, we have constructed a model of the ring-disk system for the appropriate epoch. Following Haas et al. (1982; henceforth HEMGC), the total flux from the Saturn system is

$$
\Omega_{t} B_{v}(T)=\left[\Omega_{V D}+\sum \omega_{i} e^{-\tau} \csc |B| B_{V}\left(T_{D}\right)+\sum \Omega_{i} B_{\nu}\left(T_{R}\right)\right.
$$

where $B_{v}$ is the Planck function, $\Omega_{t}$ is the total solid angle of the ring-disk system, $\Omega_{V D}$ is the solid angle of the visible disk, and $|B|$ is the ring tilt angle with respect to the Earth. The average brightness temperatures $T, T_{D}$, and $T_{R}$ are those of the system, the disk, and the rings, respectively. The $i$-th ring surface has a normal optical depth $\tau_{1}$, a visible solid angle $\Omega_{1}$, and a visible solid angle overlapping the disk $\omega_{1}$.

The rings are divided into five separate surfaces as described in HEMGC. The optical depths $\tau_{i}$ vary as $v^{\alpha} . T_{R}$ is computed from a thermometric temperature $T_{0}$ and an emissivity dependent on the $\tau_{1}$ 's (see HEMGC equation 5 and Figure 6). A fit to the data of HEMGC gives $\alpha=1.5$ 
and $T_{0}=88.7 \mathrm{~K}$ for $|B|=21.4^{\circ}$. If the $100 \mu \mathrm{m}$ brightness temperature varlation with $|B|$ has the same form as at $20 \mu \mathrm{m}$ (Nolt et al., 1980), then $T_{0}$ should be decreased by $\leqq 2 \mathrm{~K}$. This effect has been ignored since it results in a negligible change in the depth of the $\mathrm{PH}_{3}$ line.

This model for the rings was combined with our standard disk model (section IV) using equation (1). Since we are primarily concerned with the shape of the spectrum and not with its absolute callbration, we then renormalized the two nights' data to this model. This renormalization removes the difference in absolute calibration between the two data sets, forces the relative contributions of the disk and rings to be the same as in HEMGC (allowing for the changes in geometry with $|B|$ ), and ensures that the disk spectrum obtained by subtracting the ring contribution from our data will have the same normalization as our standard disk model. The renormalization factor is 0.84 for July 10 and 0.98 for July 12 .

The ring contribution was then subtracted from the measured (renormalized) spectrum using equation (1) and the ring model described above for $T_{R}$. The inferred disk spectrum for each night is shown in Figure 2 (circles) along with our standard disk model. The error bars again reflect statistical errors only. The measured contrast in the line is just over half that of the model. Increasing the contribution of the rings to the total system flux will increase the contrast in the deduced disk spectrum. For example, if we repeat the subtraction process assuming the optical depth of the rings is independent of frequency $(\alpha=0)$, then $\mathrm{T}_{\mathrm{R}}$ at $100 \mu \mathrm{m}$ increases from $63 \mathrm{~K}$ to $77 \mathrm{~K}$ (cf HEMGC's Figure 6) and the contrast in the line is increased by several kelvins (Figure 2; triangles). However, 
there is strong evidence that the brightness temperature of the rings turns over beyond 50 um (HEMGC; Daniel et al., 1982; Melnick et al., 1983). Therefore, the discrepancy between the measured line profile and our standard disk model is probably not a result of uncertainties in the ring subtraction procedure. In the following sections we only consider the disk spectrum derived assuming that $T_{R}$ decreases as in HEMGC (our case $\alpha=1.5$ ).

\section{SATURN DISK MODELS}

Disk-averaged models of Saturn were generated using the computer code described in HEMGC and Goorvitch et al. (1979). The $\mathrm{PH}_{3}$ mixing ratio was parameterized as $x=x_{0}\left(P / P_{0}\right)^{\beta}$ for $P<P_{0}$ and as $x=x_{0}$ for $P \geq P_{0}$, where $P$ is the total pressure and $\beta$ is the ratio of the dynamical scale height $\left(h_{p}=\right.$ $\mathrm{RT} / \mu \mathrm{g}$ ) to the scale helght for decreasing the $\mathrm{PH}_{3}$ mixing ratio $\mathrm{h}_{\mathrm{x}}$ (cf Encrenaz and Combes, 1977; Strobel, 1978; Tokunaga et al., 1980). Our standard model uses $P_{0}=100 \mathrm{mbar}, a \mathrm{PH}_{3}$ scale height $h_{x}=5 \mathrm{~km}$, $x_{0}=1.2 E-06=2 x_{\odot}$ (twice solar), a helium-to-hydrogen mixing ratio of $0.13 / 0.87$, an ammonia mixing ratio of $1.5 \times 10^{-4}$, and the pressure-temperature profile of Tokunaga and Cess (1977), which is very similar to that obtained by Voyager (Hanel et al., 1983).

The wavelengths for the pure rotational band of $\mathrm{PH}_{3}$ were calculated from the formula and constants given by Maki et al., (1973). Because of the lack of experimental data, the strengths were calculated using the formulas for $\mathrm{NH}_{3}$, which is a spectroscopically similar molecule. We assumed a permanent dipole moment of 0.574 Debye (Poynter and Plckett, 1980), adopted the pressure broadening data of Pickett et al. (1981), and used a Lorentzian 
line profile (cf Encrenaz et al., 1971). A $\pm 10 \%$ variation in the $\mathrm{PH}_{3}$ line strengths results in a brightness temperature change of less than $\pm 0.3 \mathrm{~K}$.

As shown in Figure 2, our standard model shows considerably more contrast in the $\mathrm{PH}_{3}$ line than is observed. We have investigated the dependence of this contrast on the parameters of the $\mathrm{PH}_{3}$ distribution $\left(\mathrm{x}_{0}\right.$, $\mathrm{P}_{0}$, and $\left.\mathrm{h}_{\mathrm{x}}\right)$, the $[\mathrm{He}] /[\mathrm{H}]$ mixing ratio, and the pressure-temperature profile. The models are relatively insensitive to the helium-to-hydrogen mixing ratio. Varying $[\mathrm{He}] /[\mathrm{H}]$ from $0.20 / 0.80$ to $0.06 / 0.94$ changes the brightness temperature in the continuum by $\$ 0.4 \mathrm{~K}$. Since the core of the line is formed near the inversion ( $-70 \mathrm{mbar}$ ), an inversion temperature of $-93 \mathrm{~K}$ is required to reduce the model contrast to that which is observed. Such variations in the pressure-temperature profile are inconsistent with past observations, including the Voyager IRIS experiment (Hanel et al., 1983) and the radio occultation mean (Tyler et al., 1982).

The brightness temperature in the core of the line roughly corresponds to the atmospheric level where the optical depth reaches unity. For a given pressure-temperature profile, the most straightforward way to raise the brightness temperature in the core is to either increase or decrease the opacity such that $\tau=1$ lies above or below the thermal inversion. Figure 3 shows the effect of decreasing $\mathrm{P}_{0}$, the pressure above which the $\mathrm{PH}_{3}$ mixing ratio falls exponentially, from 100 to 10 mbar. Such models place significant amounts of $\mathrm{PH}_{3}$ in the stratosphere, producing a strong emission line core. Note that the data points at 102.41 and 102.92 um lie near the minimum brightness temperature in the manifold, whereas the data point at $102.78 \mu \mathrm{m}$ lies near the maximum of the emission core. These models are 
inconsistent with the observations, which show a similar brightness temperature at all three wavelengths. The strength of the emission core is reduced somewhat if we use the pressure-temperature profile deduced from Voyager 2 radio occultation measurements (Tyler et al., 1982), because it has significantly cooler temperatures above the inversion (cf Orton, 1983). However, such models still produce a substantial emission core which is inconsistent with the present observations.

Varying the scale height $h_{x}$ gives results similar to those in Figure 3 . That is, increasing $h_{x}$ to $10 \mathrm{~km}$ puts more $\mathrm{PH}_{3}$ above the tropopause and produces an emission line core similar to the model with $P_{0}=50$ mbar. From the present observations we therefore conclude that $\mathrm{PH}_{3}$ must be strongly depleted above the inversion. Tokunaga et al. (1980) and Winkelstein et al. (1983) arrive at similar conclusions based on observations in the near infrared and the ultraviolet, respectively. However, Courtin et al. (1984) find evidence in the Voyager IRIS spectra at $10 \mu \mathrm{m}$ for a uniform mixing ratio of $5 X$ solar to an altitude of 3 to 5 mbar. Prinn et al. (1984) suggest that this discrepancy may result from the nonunlqueness of the thermal inversion process or to latitudinal or seasonal changes in the $\mathrm{PH}_{3}$ vertical profile. Theoretical models predict that phosphine should be strongly depleted in the stratosphere because of photolysis by ultraviolet radiation (Prinn and Lewis, 1975; Strobel, 1977; 1978). Similar depletions have been observed on Juplter (e.g., Tokunaga et al., 1979; Kunde et al.. 1982).

An acceptable fit to the present observations can be obtained if a stratospheric "cloud" or haze is included in our standard model with an 
optical depth of 0.25 at $100 \mu \mathrm{m}$. In such a model the $\mathrm{PH}_{3}$ manifold is still formed above the inversion, but without a significant emission core. However, it may be difficult to make particles large enough to absorb at $100 \mu \mathrm{m}$ and still small enough to remain buoyant at pressures $\$ 100 \mathrm{mbar}$ (Orton, 1983). Moreover, there is little independent evidence for the existence of large stratospheric particles in other spectral bands (Tomasko et al. 1984).

As discussed above, the brightness temperature in the $\mathrm{PH}_{3}$ manifold can also be raised by forming the line below the inversion. In Figure 4 we show the results of varying $x_{0}$, the uniform mixing ratio assumed for $P \geqq P_{0}$. The manifold becomes increasingly narrow as the mixing ratio is decreased. However, the brightness temperature in the core remains nearly constant until the mixing ratio is sufficiently small $\left(x_{0} \leq 0.05 x_{\odot}\right)$ to permit formation of the core $(\tau-1)$ below the inversion at higher temperatures. Such models are able to fit the observed core of the manifold, but require $x_{0} \ll x_{\odot}$, which is inconsistent with the near and middle infrared observations which find $x_{0}-2-5 x_{\odot}$ in the lower troposphere (Tokunaga et al., 1980; Larson et al., 1980; Courtin et al., 1984).

Since the near and middle infrared vibrational transitions probe deeper into the troposphere (cf Encrenaz and Combes, 1980), a consistent model can be obtained by keeping $x_{0} \geqq 2 x_{\odot}$ and increasing $P_{0}$. Such models maintain a large abundance of $\mathrm{PH}_{3}$ in the lower troposphere, but show substantial depletions in the vicinity of the tropopause. Figure 5 shows the effect of increasing $P_{0}$ from 100 mbar to 1 bar. The model with $P_{0}=500$ mbar provides an excellent fit to our measured profile. 
Moreover, the inferred phosphine distribution is remarkably similar to that preferred by Kaye and Strobel (1984), who consider the detalled photochemistry of Saturn's atmosphere and derive $\mathrm{PH}_{3}$ distributions for different values of the eddy diffusion coefficient (K). Our best fitting distribution $\left(P_{0}=500\right.$ mbar, $h_{x}=5 \mathrm{~km}$, and $\left.x_{0}=2 x_{\odot}\right)$ closely corresponds to the one they calculate for $K=10^{4} \mathrm{~cm}^{2} \mathrm{sec}^{-1}$ in the upper troposphere. Similar values of $K$ are inferred from observations of ortho and para hydrogen on the Jovian planets (Massie and Hunten, 1982).

\section{CONCLUSIONS}

The main conclusions of this paper are:

(1) The detection of the far-infrared $\mathrm{J}=10$ pure rotational manifold of phosphine $\left(\mathrm{PH}_{3}\right)$ on Saturn has been confirmed,

(2) The lack of a significant emission core in this manifold implies that there is little or no $\mathrm{PH}_{3}$ above the inversion, and

(3) The most straightforward explanation for the relatively low brightness temperature contrast in the $J=10$ manifold is a significant depletion of $\mathrm{PH}_{3}$ in the upper troposphere $\left(\mathrm{P}_{0} \leqq 500 \mathrm{mbar}\right)$. This suggests that the eddy diffusion coefficient in Saturn's troposphere is $-10^{4} \mathrm{~cm}^{2} \mathrm{sec}^{-1}$.

Improved sampling of the individual manifolds, as well as observations of several additional manifolds, would be useful in further elucidating the detalls of the $\mathrm{PH}_{3}$ distribution in the vicinlty of the tropopause. 


\section{ACKNOWLEDGEMENTS}

We particularly wish to thank E. Bekstrom for his technical support, P. B. Duffy for his work on the flight series and on the detector development, and J. P. Simpson for her assistance with the observations. We are grateful to P. Viscuso for discussing his observations prior to publication and to $R$. Courtin for a helpful discussion regarding the phosphine pressure broadening coefficients. As always, it is a pleasure to acknowledge the staff of the Kuiper Airborne Observatory for their continuing support. Partial support for this research was provided by the NASA Headquarters Office of Astronomy and Astrophysics. 


\section{REFERENCES}

Barshay, S., and J. S. Lewis (1978). Chemical structure of the deep atmosphere of Jupiter. Icarus $\underline{33}, 593-611$.

Courtin, R., D. Gautier, A. Marten, B. Bezard, and R. Hanel (1984). The composition of Saturn's atmosphere at northern temperate latitudes from Voyager IRIS spectra: $\mathrm{NH}_{3}, \mathrm{PH}_{3}, \mathrm{C}_{2} \mathrm{H}_{2}, \mathrm{C}_{2} \mathrm{H}_{6}, \mathrm{CH}_{3} \mathrm{D}, \mathrm{CH}_{4}$, and the Saturnian D/H isotopic ratio. Astrophys. J. 287, 899-916.

Daniel, R. R., S. K. Ghosh, K. V. K. Iyengar, T. N. Rengarajan, S. N. Tandon, and R. P. Verma (1982). Far-infrared brightness temperature of Saturn's disk and rings. Icarus 49, 205-212.

Encrenaz, Th., D. Gautler, and L. Vapilion (1971). The far infrared spectrum of Jupiter. Astron. Astrophys. 11, 431-449.

Encrenaz, Th. and M. Combes (1977). The far infrared spectrum of Saturn: observability of $\mathrm{PH}_{3}$ and $\mathrm{NH}_{3}$. Astron. Astrophys. 61, 387-390.

Encrenaz, Th. and M. Combes (1980). The composition of planetary atmospheres, Infrared Astronomy, The Proceedings of IAU Symposium No. 96, Edited by C. G. Wynn-Williams and D. P. Cruikshank, D. Reidel Publishing Company, Boston, pp. 1-33.

Erickson, E. F., S. Matthews, G. C. Augason, J. R. Houck, D. M. Rank, and M. R. Haas (1984a). All-aluminum optical system for a large cryogenically cooled far infrared echelle spectrometer. Proc. Soc. Photo-Optical Instrumentation Engineers 509, 129-139. 
Erlckson, E. F., J. R. Houck, M. O. Harwit, D. M. Rank, M. R. Haas, D. J. Hollenbach, J. P. Simpson, G. C. Augason, and D. D. MoKibbin (1984b). A far infrared echelle spectrometer for the Kuiper Airborne Observatory. Symposium on Airborne Astronomy, NASA Conference Publication 2353, H. Thronson and E. Erickson, editors, pp. 313-319.

Erickson, E. F., J. R. Houck, M. O. Harwit, D. M. Rank, M. R. Haas, D. J. Hollenbach, J. P. Simpson, and G. C. Augason (1985). A FIR cooled grating spectrometer for the Kuiper Airborne Observatory. Infrared Physics 25, 513-515.

Gautier, D., and T. Owen (1983). Cosmogonical implications of elemental and isotropic abundances in atmospheres of the giant planets. Nature 304 , $691-694$.

Goorvitch, D., E. F. Erickson, J. P. Simpson, and A. T. Tokunaga (1979). The mean Jovian temperature structure derived from spectral observations from 105 to $630 \mathrm{~cm}^{-1}$. Icarus $40,75-86$.

Haas, M. R., E. F. Erickson, D. D. Mckibbin, D. Goorvitch, and L. J. Caroff (1982). Far-infrared spectrophotometry of Saturn and its rings. Icarus $51,476-490$.

Haas, M. R., E. F. Erickson, D. Goorvitch, D. D. McKibbin, and D. M. Rank (1984). Detection of the $J=10$ manifold of the pure rotational band of phosphine on Saturn. Symposium on Airborne Astronomy, NASA Conference Publication 2353, H. Thronson and E. Erickson, editors, pp. $76-80$. 
Hanel, R., B. Conrath, F. M. Flasar, V. Kunde, W. Maguire, J. Pearl,

J. Pirraglia, R. Samuelson, L. Herath, M. Allison, D. Cruikshank, D. Gautier, P. Gierasch, L. Horn, R. Koppany, and C. Ponnamperuma (1981). Infrared observations of the Saturnian system from Voyager 1. Science $212,192-200$.

Hanel, R. A., B. J. Conrath, V. G. Kunde, J. C. Pearl, and J. A. Pirraglia (1983). Albedo, internal heat flux, and energy balance of Saturn. Icarus $\underline{53}, 262-285$.

Kaye, J. A., and D. F. Strobel (1984). Phosphine photochemistry in the atmosphere of Saturn. Icarus 59, 314-335.

Kunde, V., R. Hanel, W. Maguire, D. Gautier, J. P. Baluteau, A. Marten, A. Chedin, N. Husson, and N. Scott (1982). The tropospheric gas composition of Jupiter's north equatorial belt $\left(\mathrm{NH}_{3}, \mathrm{PH}_{3}, \mathrm{CH}_{3} \mathrm{D}, \mathrm{GeH}_{4}\right.$, $\mathrm{H}_{2} \mathrm{O}$ ) and the Jovian $\mathrm{D} / \mathrm{H}$ isotopic ratio. Astrophys. J. 263, 443-467.

Larson, H. P., U. Fink, H. A. Smith, and D. S. Davis (1980). The middle-infrared spectrum of Saturn: Evidence for phosphine and upper limits to other trace atmospheric constituents. Astrophys. J. 240, $327-337$.

Lewis, J. S. (1969). Observability of spectroscopically active compounds in the atmosphere of Jupiter. Icarus 10, 393-409.

Maki, A. G., R. L. Sams, and Wm. B. Olson (1973). Infrared determination of $C_{0}$ for phosphine via perturbation-allowed $\Delta|k-1|= \pm 3$ transitions in the $3 v_{2}$ band. J. Chem. Physics 58, 4502-4512. 
Massie, S. T., and D. M. Hunten (1982). Conversion of para and or tho hydrogen in the Jovian planets. Icarus $49,213-216$.

Matthews, S., and E. F. Erickson (1977). Analytical calculation of the areas of Saturn's disk and rings. NASA TM X-73,204.

Melnick, G., R. W. Russell, T. R. Gosnell, and M. Harwit (1983). Spectrophotometry of Saturn and its rings from 60 to 180 microns. Icarus $53,310-318$.

Nolt, I. G., E. W. Barrett, J. Caldwell, F. C, Gillett, R. E. Murphy, J. V. Radostitz, and A. T. Tokunaga (1980). IR brightness and eclipse cooling of Saturn's rings. Nature 283, 842-843.

Orton, G. S. (1983). Thermal infrared constraints on ammonia lce particles as candidates for clouds in the atmosphere of Saturn. Icarus $\underline{53}$, 293-300.

Pickett, H. M., R. L. Poynter, and E. A. Cohen (1981). Pressure broadening of phosphine by hydrogen and helium. J. Quant. Spectrosc. Radiat. Transfer $26,197-198$.

Poynter, R. L., and H. M. Pickett (1980). Submillimeter, millimeter, and microwave spectral line catalogue. Jet Propulsion Laboratory Publ. 80-23, Pasadena, California.

Prinn, R. G., and J. S. Lewis (1975). Phosphine on Jupiter and implications for the great red spot. Science 190, 274-276.

Prinn, R. G., H. P. Larson, J. J. Caldwell, and D. Gautier (1984). 
Composition and chemistry of Saturn's atmosphere. Saturn, University of Arizona Press, Tucson, AZ, T. Gehrels and M. S. Matthews, editors, pp. 88-149.

Simpson, J. P., J. N. Cuzzi, E. F. Erickson, D. W. Strecker, and

A. T. Tokunaga (1981). Mars: Far Infrared spectra and thermal emission models. Icarus $48,230-245$.

Strobel, D. F. (1977). $\mathrm{NH}_{3}$ and $\mathrm{PH}_{3}$ photochemistry in the Jovian atmosphere. Astrophys. J. (Letters) 214, L97-L99.

Strobel, D. F. (1978). Aeronomy of Saturn and Titan. The Saturn System, NASA Conference Publication 2068, D. H. Hunten and D. Morrison, editors, pp. 185-194.

Tokunaga, A., and R. D. Cess (1977). A model for the temperature inversion within the atmosphere of Saturn. Icarus $32,321-327$.

Tokunaga, A. T., R. F. Knacke, S. T. Ridgway, and L. Wallace (1979). High-resolution spectra of Jupiter in the range 744-980 inverse centimeter spectral range. Astrophys. J. 232, 603-615.

Tokunaga, A. T., H. L. Dinerstein, D. F. Lester, and D. M. Rank (1980). The phosphine abundance on Saturn derived from new 10-micrometer spectra. Icarus $42,79-85$.

Tomasko, M. G., R. A. West, G. S. Orton, and V. G. Tejfel (1984). Clouds and aerosols in Saturn's atmosphere. Saturn, University of Arizona Press, Tucson, AZ, T. Gehrels and M. S. Matthews, editors, pp. 150-194. 
Tyler, G. L., V. R. Eshleman, J. D. Anderson, G. S. Levy, G. F. Lindal, G. E. Wood, and T. A. Croft (1982). Radio science with Voyager 2 at Saturn: Atmosphere and Ionosphere and the masses of Mimas, Tethys, and Iapetus. Science 215, 553-557.

Vohden, R. A., and A. Boksenberg (1984). The Astronomical Almanac. pp. E66, E82, and F41.

Winkelstein, P., J. Caldwell, S. J. Kim, M. Combes, G. E. Hunt, and V. Moore (1983). A determination of the composition of the Saturnian stratosphere using the IUE. Icarus 54, 309-318. 
Table 1

Brightness Temperatures for Saturn

\begin{tabular}{ccc} 
Date & $\begin{array}{c}\text { Wavelength } \\
(\mu \mathrm{m})\end{array}$ & $\begin{array}{c}\text { Brightness } \\
\text { Temperature }(\mathrm{K})\end{array}$ \\
\hline 1984 July 10 & 97.84 & $91.2 \pm 0.3$ \\
& 102.78 & $85.5 \pm 0.3$ \\
& 102.92 & $86.4 \pm 0.3$ \\
& 104.83 & $91.6 \pm 0.7$ \\
& 97.84 & $84.9 \pm 0.6$ \\
& 102.41 & $81.6 \pm 0.6$ \\
& 102.78 & $80.3 \pm 0.5$ \\
& 102.92 & $81.0 \pm 0.3$ \\
& 103.52 & $83.6 \pm 0.3$ \\
& 104.83 & $84.4 \pm 0.3$
\end{tabular}




\section{FIGURE CAPTIONS}

Figure 1. (a) The atmospheric transmission at $12.5 \mathrm{~km}$ for 10 precipitable microns of water (typical) at a resolution of $\lambda / \Delta \lambda=5000$. (b) The measured brightness temperatures of the ring-disk system of Saturn (circles are for July 10; triangles are for July 12). The error bars represent statistical errors only.

Figure 2. The circles represent the data of Figure 1 with the contribution of the rings removed assuming their optical depth falls off as $\nu^{1.5}$ (cf HEMGC). The triangles are the same data with the contribution of the rings removed assuming their optical depth is independent of frequency $(\alpha=0)$. The solid curve shows our standard disk-averaged model of Saturn at the same resolution.

Figure 3. The data are from Figure 2 (circles are July 10; triangles are July 12) with the ring component removed assuming $\alpha=1.5$. The curves are disk models with different values of $P_{0}$, the pressure level below which the $\mathrm{PH}_{3}$ mixing ratio is constant rather than exponential. As $\mathrm{P}_{0}$ decreases, the amount of stratospheric $\mathrm{PH}_{3}$ increases. The model with $\mathrm{P}_{0}=100$ mbar is the standard model described in the text. Recall that the data have been normalized to force the two continuum points to fit this model.

Figure 4. The data are the same as in Figure 3. The models have different values of $x_{0}$, the (uniform) mixing ratio for $P \leqq P_{0}=100$ mbar. ranging from 0.04 to 4 times solar.

Figure 5. The data are the same as in Figure 3. The models have different values of $P_{0}$, all at or below the tropopause. 


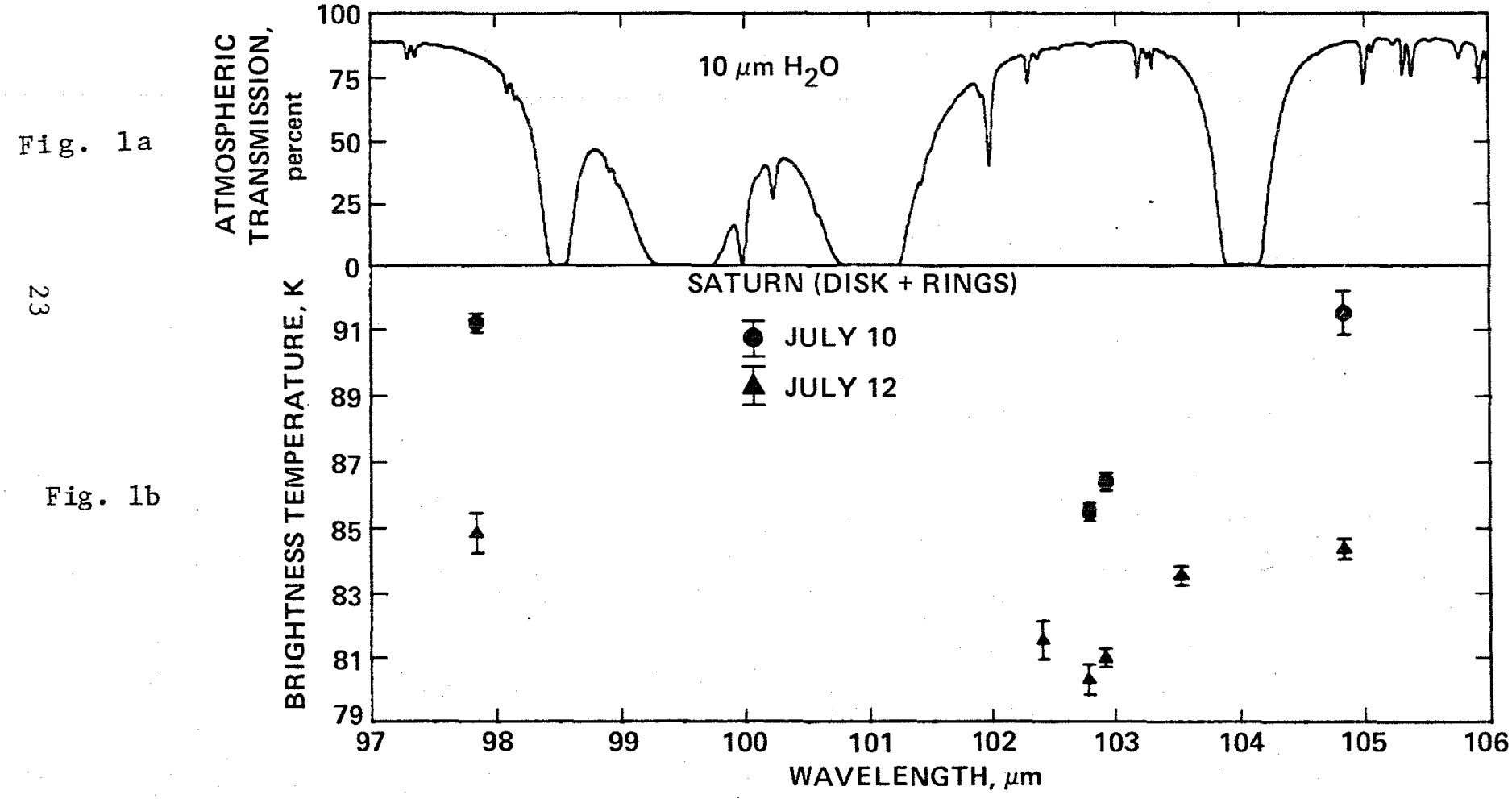

iig. 1a,1b 


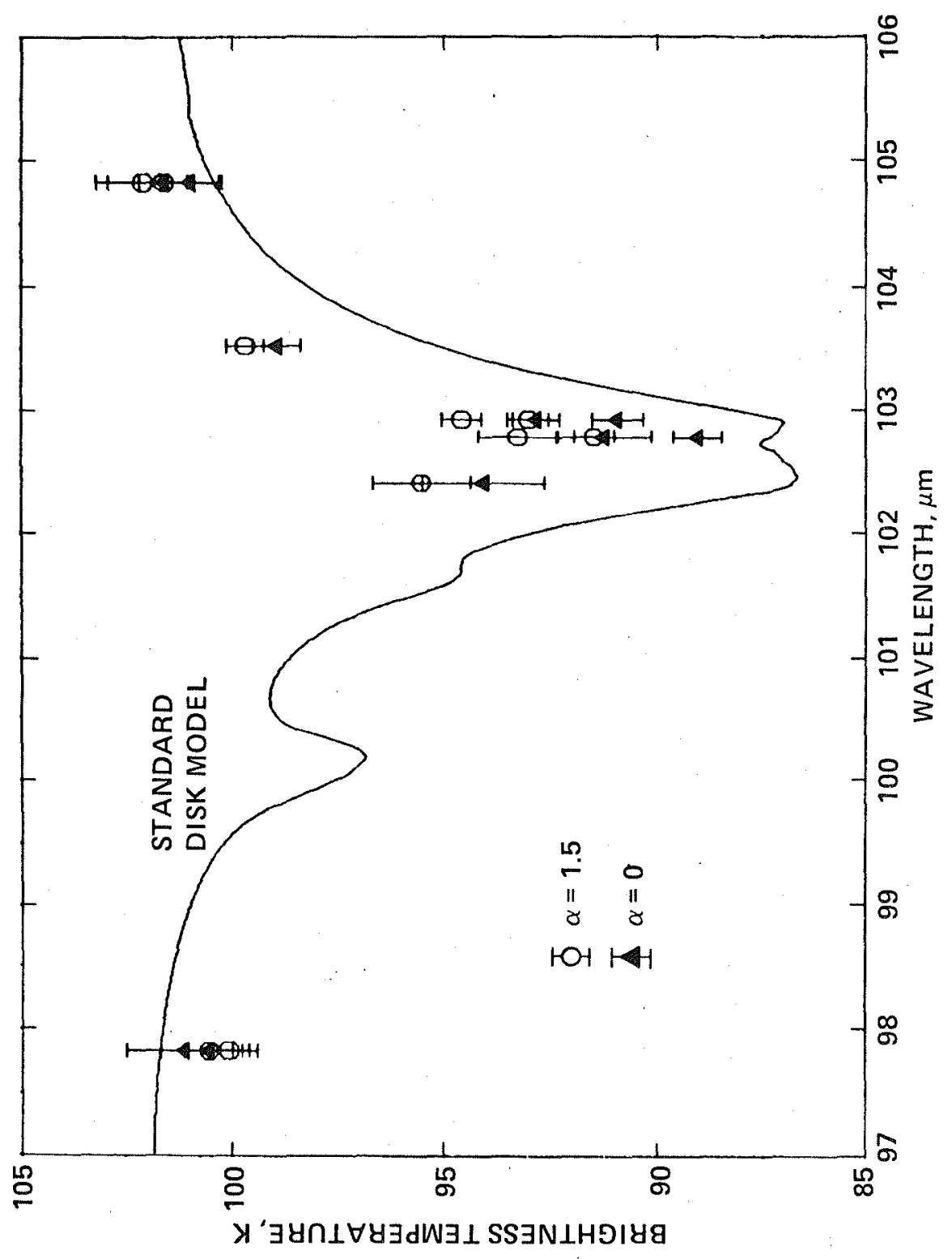

Fig. 2 


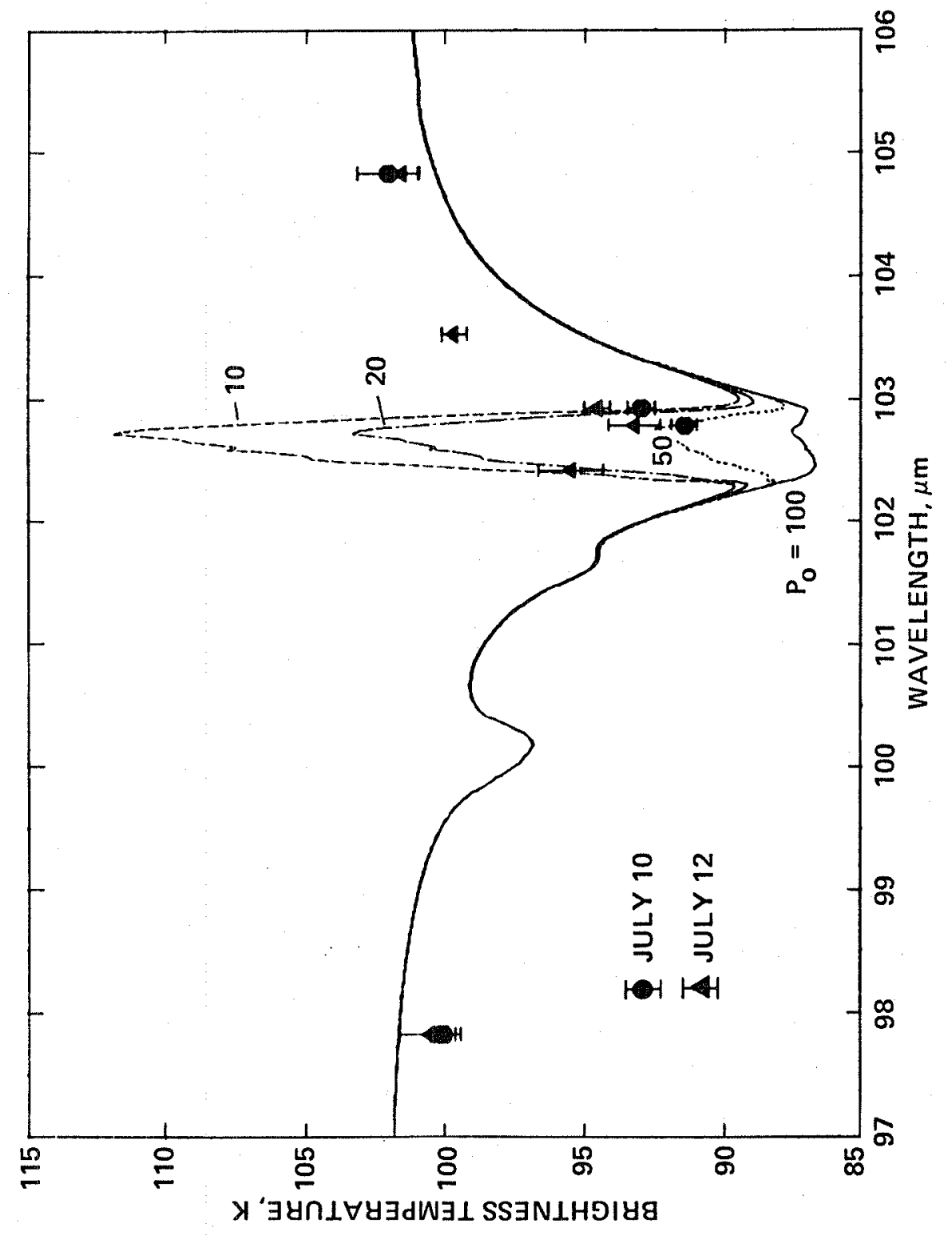

Fig. 3 


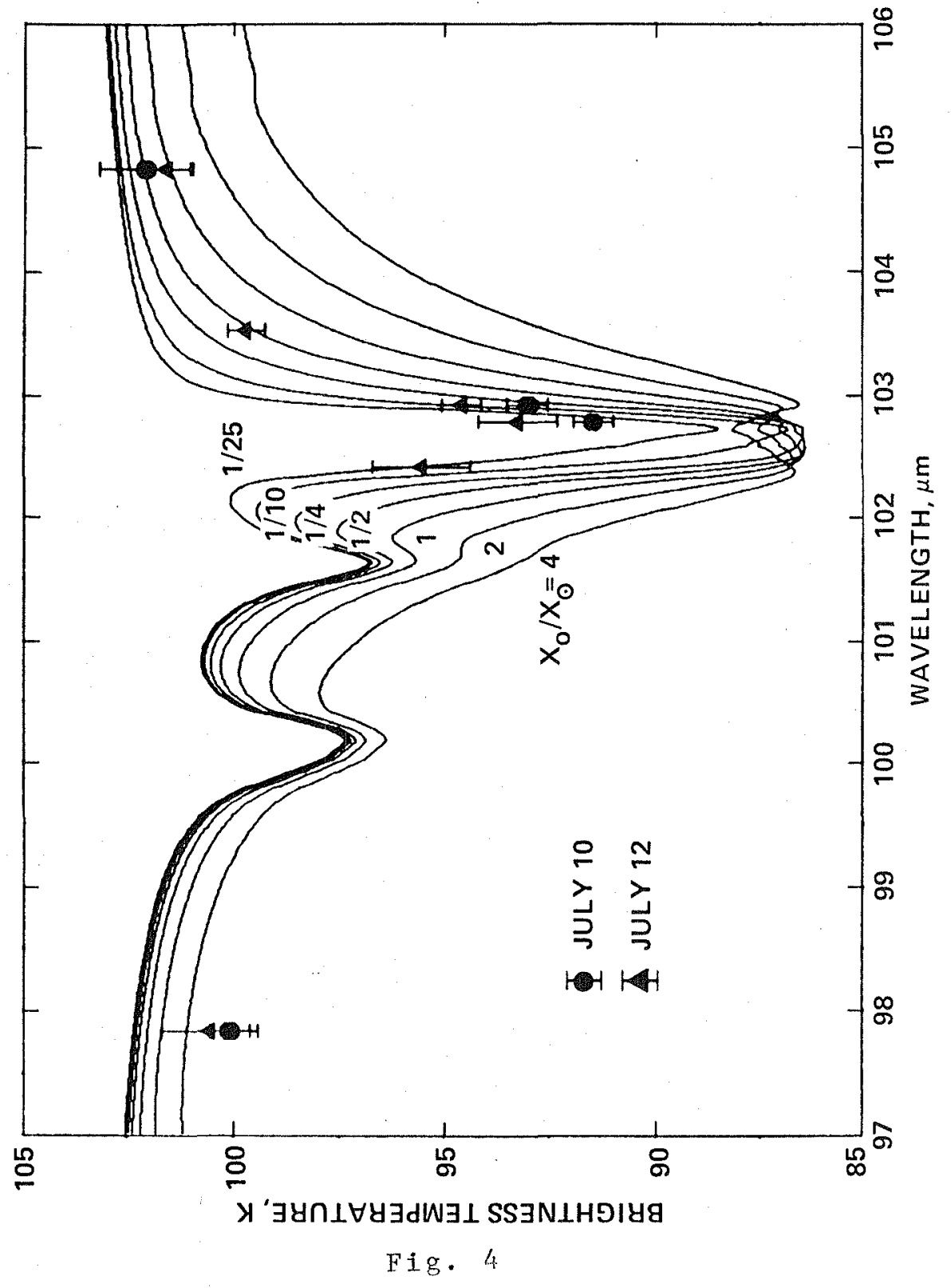




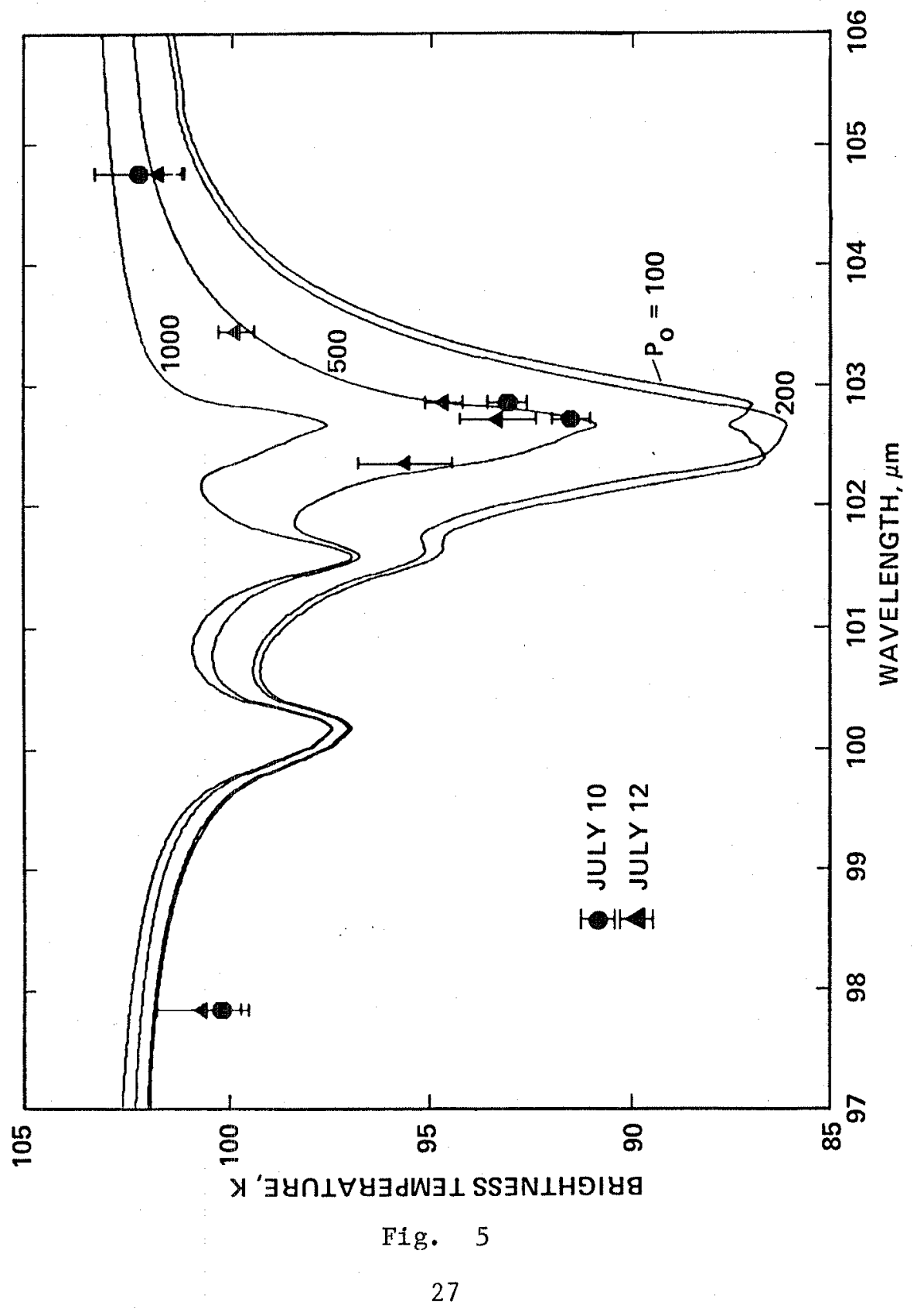




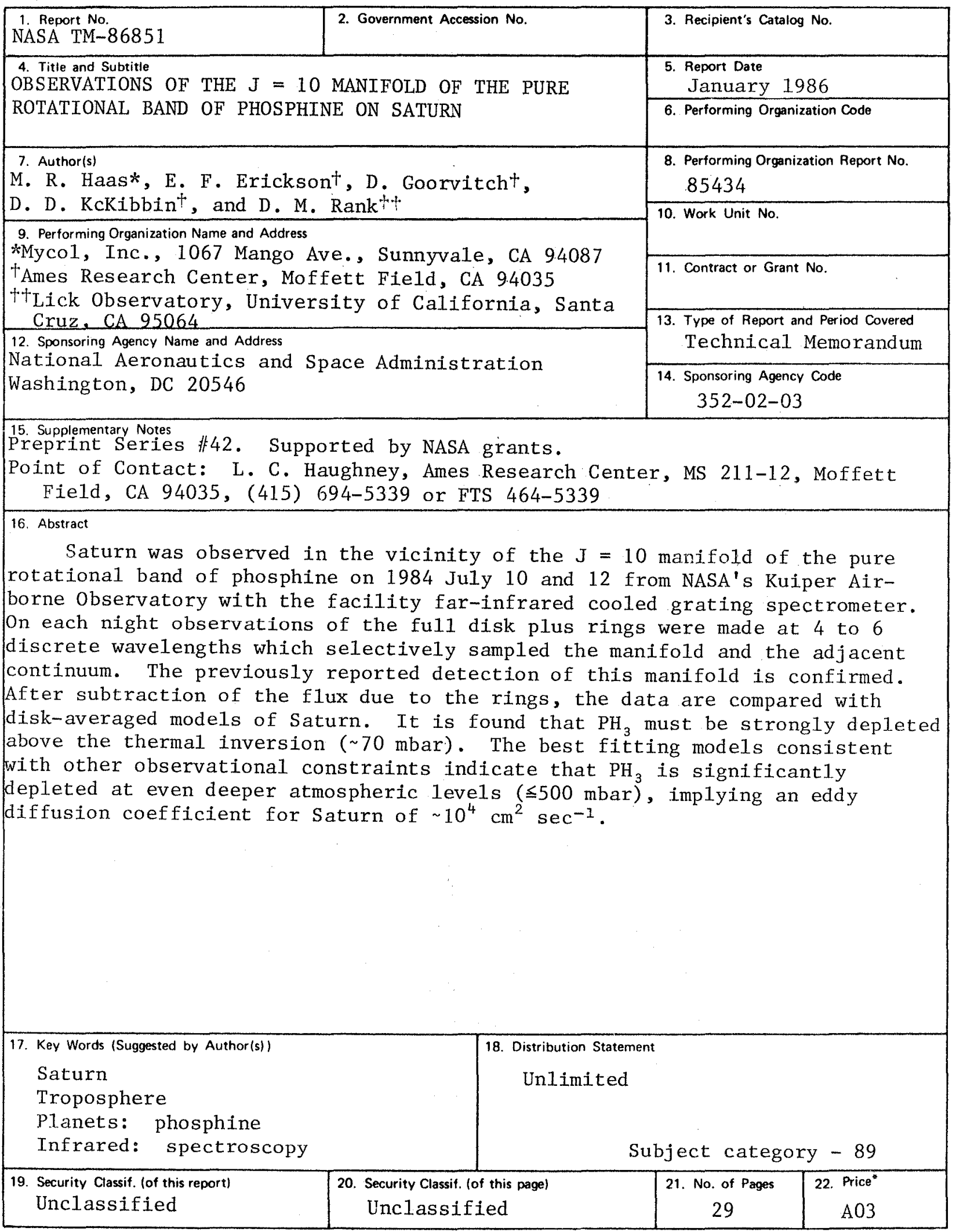

"For sale by the National Technical Information Service, Springfield, Virginia 22161 
End of Document 